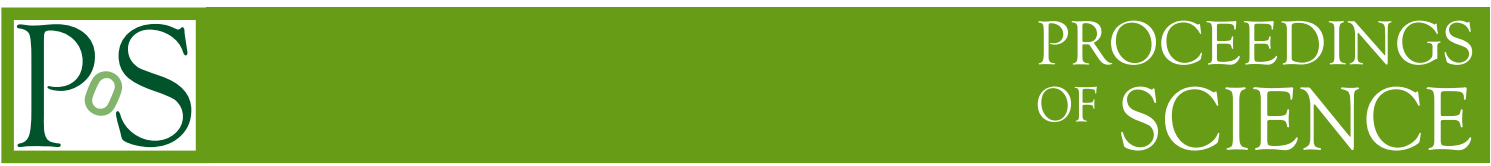

\title{
Charged track reconstruction and $b$-tagging performance in ATLAS
}

\author{
Andrea Favareto* \\ Università degli Studi di Milano and INFN Milano \\ E-mail: andrea.favareto@mi.infn.it
}

The ATLAS Inner Detector is designed to provide precision tracking information at LHC luminosities with a hermetic detector covering 5 units in pseudo-rapidity. It features a large silicon tracker subdivided into a pixel and a strip system for precise tracking and primary/secondary vertex reconstruction and to provide excellent $b$-tagging capabilities. A Transition Radiation Tracker improves the momentum reconstruction and provides electron identification information.

The subject of these proceedings is the performance of the ATLAS Inner Detector achieved after its first 2 years of operation. The excellent detector performance and more than a decade of simulation studies provided a good basis for the commissioning of the offline track and vertex reconstruction. Early studies with cosmic events and the ever increasing amount of high quality p-p collision data allowed for rapid progress in understanding of the detector. Today the ATLAS Inner Detector approaches its design values in most relevant performance criteria. High luminosity pileup and tracking in central heavy ion events pose additional challenges. An overview of the recent tracking performance results and the lessons learned during commissioning will be given, including a brief overview of the main $b$-tagging algorithms and its performances.

LHC on the March,

November 16-18, 2011

Protvino, Moscow region, Russian Federation

*Speaker.

$\dagger$ On behalf of the ATLAS Collaboration 


\section{Introduction}

The Large Hadron Collider [1] is a particle physics accelerator built at CERN. It collides head-on bunches of protons or heavy ions. The analysis of these collisions may help to discover the Standard Model (SM) Higgs boson or new physics phenomena beyond the SM.

The ATLAS experiment [2] is a general purpose experiment that records collision events produced by the LHC. In order to fulfill its scientific objectives, ATLAS is equipped with an Inner Detector tracking system embedded in a $2 \mathrm{~T}$ axial magnetic field surrounding the interaction point. The calorimetry system is located beyond the ID solenoid and combines two techniques: liquid Argon and sampling technique with plastic scintillators embedded in an iron absorber. Finally a muon detector system which occupies the outermost region of the detector and uses an air-core toroid with strong bending power in a large volume within a light and open structure.

In the coordinate system used in ATLAS the nominal interaction point is defined as the origin of the coordinate system, while the beam direction defines the $z$-axis and the $(x, y)$ plane is transverse to the beam direction. The positive $x$-axis is defined as pointing from the interaction point to the center of the LHC ring and the positive $y$-axis is defined as pointing upwards. The side-A of the detector is defined as that with positive $z$ and side-C is that with negative $z$. The azimuthal angle $\phi$ is measured as usual around the beam axis, and the polar angle $\theta$ is the angle from the beam axis.

The LHC started its operations late 2009, by colliding proton beams at $900 \mathrm{GeV}$ center of mass energy. During 2010 and 2011 the LHC has produced proton-proton collisions at $7 \mathrm{TeV}$. The integrated luminosity recorded by ATLAS amounts to approximately $40 \mathrm{pb}^{-1}$ in 2010 , whilst more than $5 \mathrm{fb}^{-1}$ of data were already collected during 2011 at the time of writing. LHC also did collide in 2010 and in 2011 heavy ions (lead). Such collisions produce far more tracks than the $7 \mathrm{TeV}$ proton-proton collisions and they do represent a challenge for the ATLAS Inner Detector tracking system.

This contribution summarizes the tracking, vertexing and $b$-tagging performance of the ATLAS Inner detector.

\section{The ATLAS Inner Detector}

Tracks and vertices are the basic input for most physics measurements, therefore ATLAS has a sophisticated tracking system at the center, around the interaction point. The ATLAS Inner Detector consists of three different sub-detectors which can be seen schematically in Fig.1.

A superconducting solenoid surrounds the entire tracking system and produces a $2 \mathrm{~T}$ axial magnetic field along the beam axis direction. This magnetic field bends the trajectory of the charged particles which allows to measure their momentum.

The innermost part of the ATLAS Inner Detector is the Pixel Detector. It consists of $400 \mu \mathrm{m} \times$ $50 \mu \mathrm{m}$ pixels and is arranged in three barrel layers with end-caps on each side of the detector. Typically three hits are expected for a track passing through all barrel layers. The Pixel Detector is surrounded by the Semi-Conductor Tracker Detector (SCT) which uses silicon micro-strips to increase the number of space points available for track measurements. The SCT Detector is arranged in four barrel layers with end-caps. All the layers consist of double-sided modules with a $40 \mathrm{mrad}$ stereo angle to provide the space-point measurements. Eight hits are expected for a track crossing 


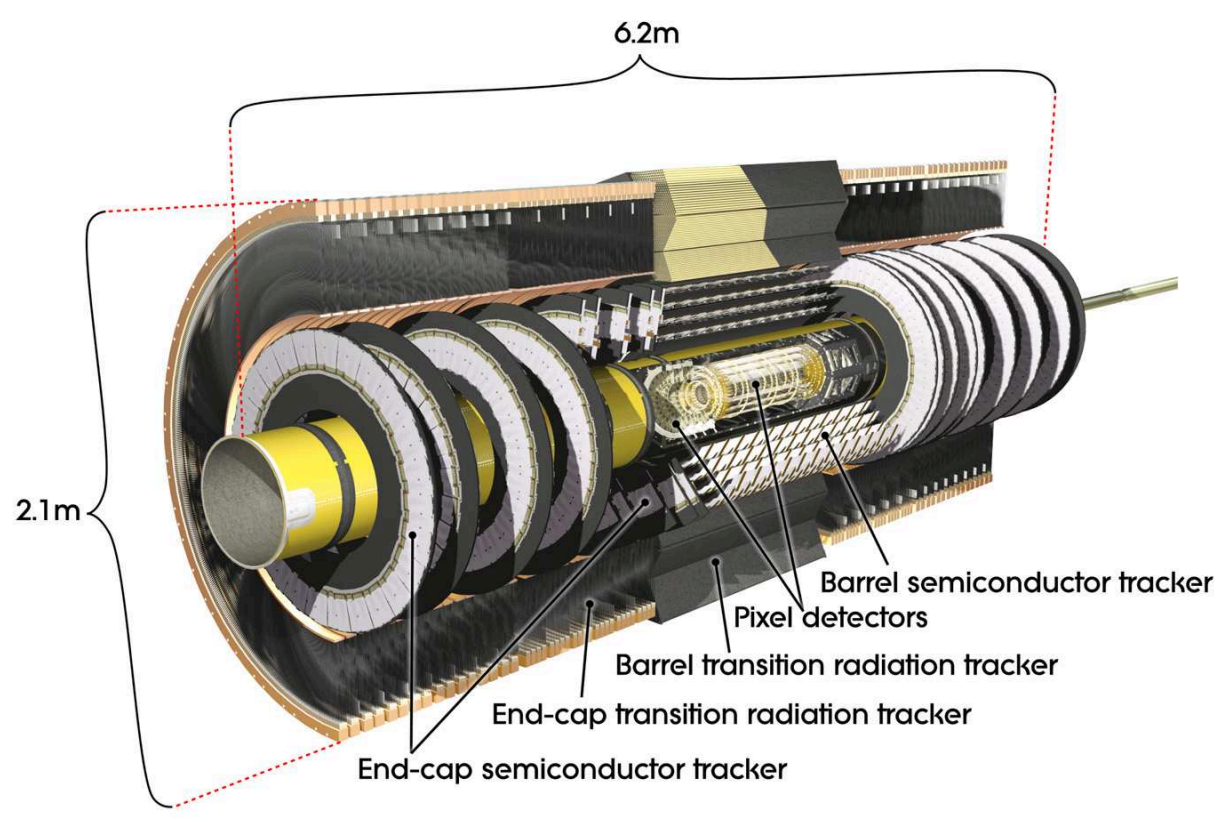

Figure 1: Schematic view of the Inner Detector

all layers. The Transition Radiation Tracker (TRT) consists of $4 \mathrm{~mm}$ diameter straw tubes to extend the track measurements with about 30 hits for an average track. It is also capable of identifying electrons through their transition radiation.

\section{Track Reconstruction}

Reconstruction of tracks and measurement of their parameters is mandatory for most physics applications. In particular, the impact parameters and momentum of charged particles have to be measured with high precision. Tracks are reconstructed within the full ID acceptance range $(|\eta|<2.5)$ using a $\chi^{2}$ fitter. The pattern recognition [3] works primarily inside-out, associating first the silicon hits and then extrapolating to the TRT to include its measurements. Though there is also an outside-in tracking, which uses the TRT track segments seeds plus and inward extension in order to efficiently reconstruct tracks with secondary interactions. The pattern recognition selects first very loose track candidates and then a stringent ambiguity processor selects good track candidates (Fig. 2). Only those satisfying a minimum number of silicon hits and a cut in the transverse and longitudinal impact parameter with respect to the beam spot are kept. The tracking has been performed down to $p_{T}$ as low as $100 \mathrm{MeV}$ [4] with efficiency shown in Fig. 3.

Heavy ion conditions give also the opportunity to study tracking under high occupancy conditions, comparable to the ones expected in future high luminosity LHC updates. In this conditions tighter requirement need to be applied [5].

\section{Vertex Reconstruction}

Measured tracks are used to reconstruct the interaction point and secondary vertices from 


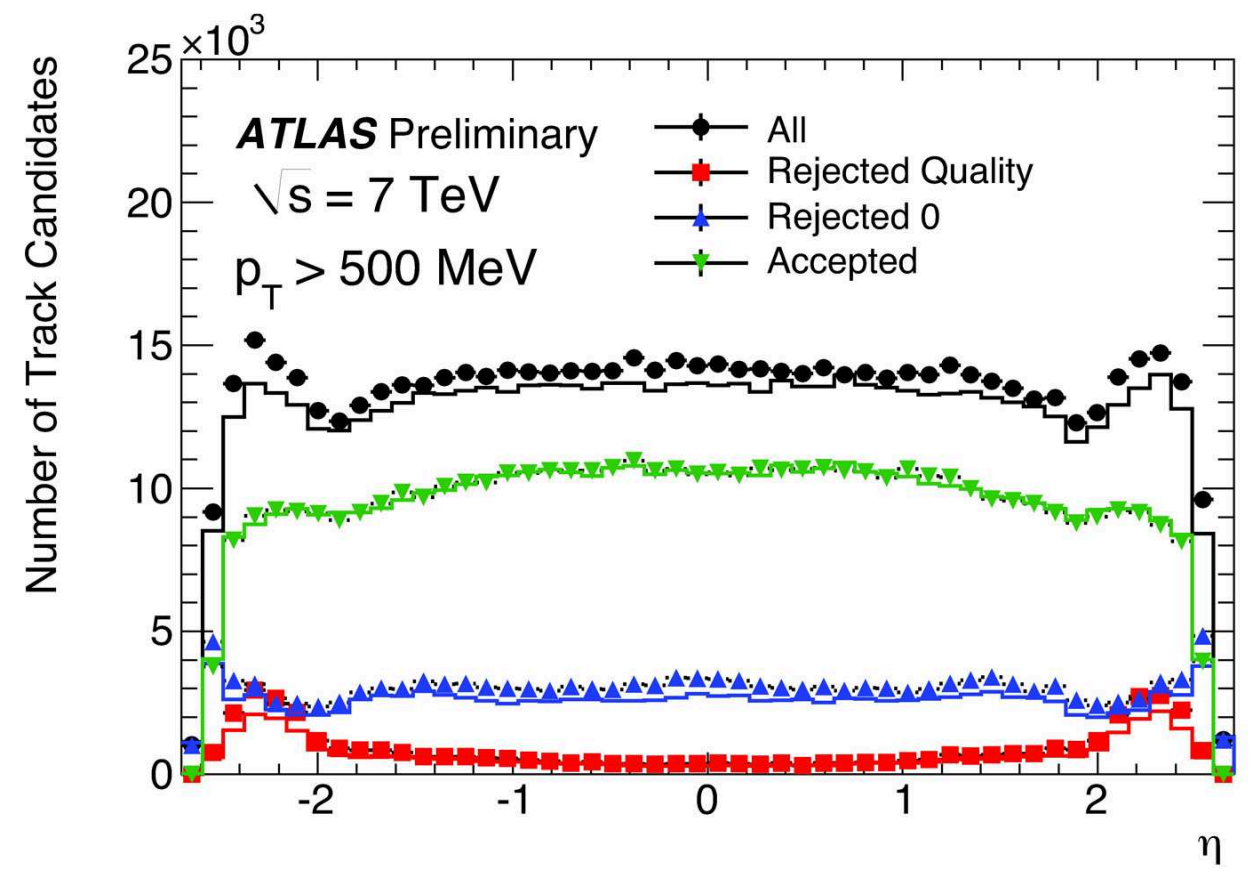

Figure 2: Number of track candidates as a function of $\eta$ and at different stages of the track finding. All track seeds are plotted in black and the resolved tracks in green. Data are compared with simulation.
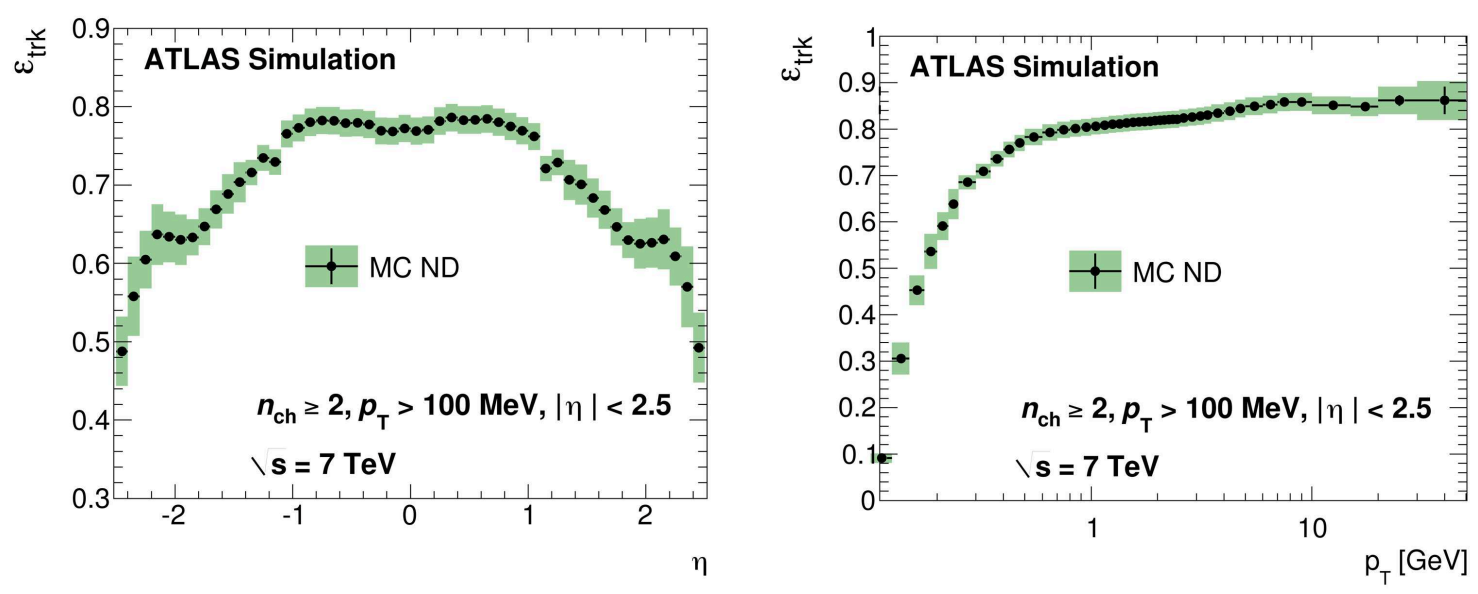

Figure 3: Track reconstruction efficiency as a function of $\eta$ (left) and of $p_{T}$ (right) derived from nondiffractive MC.

particle decays and interactions with detector material. Excellent performance of the vertex reconstruction is mandatory for many applications like $b$-tagging (see section 7) or the identification and rejection of pile-up, where many $p p$ collisions occur in the same beam crossing. This is especially relevant at the high peak luminosity of $3.65 \times 10^{33} \mathrm{~cm}^{-2} \mathrm{~s}^{-1}$ reached in 2011 which corresponds in average to 17 interactions per crossing.

The ATLAS vertex reconstruction works using an iterative algorithm [6]: first a vertex finding is executed where tracks are associated to a primary vertex and in the second stage the vertex is 

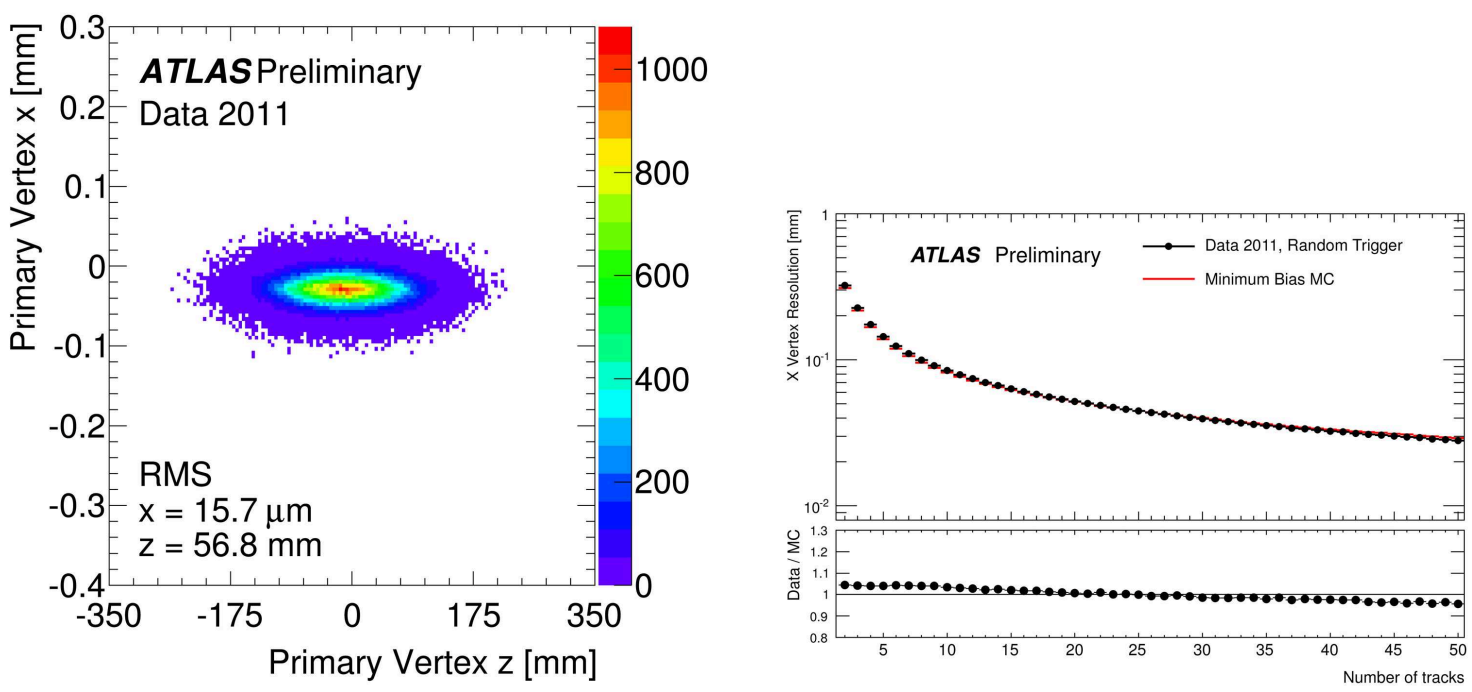

Figure 4: Two dimensional distribution of reconstructed primary vertices in the $(x, z)$ plane (left), and the estimated vertex resolution $\sigma_{X}$ as a function of tracks per vertex (right).

fitted (with a $\chi^{2}$ technique). The vertex fitter include a beam-spot constraint, which is routinely online and offline computed. It is approximatively $15 \mu \mathrm{m}$ in the transverse plane, and $5.7 \mathrm{~mm}$ in the longitudinal plane as shown in Fig. 4. The primary vertex resolution is extracted using a data driven method (mainly split vertex technique). It is about $23 \mu \mathrm{m}$ on the transverse plain and about $40 \mu \mathrm{m}$ on the longitudinal plane for vertices with 70 tracks. It depends on the number of tracks as well as on the $\sqrt{\sum_{\operatorname{trk}} p_{T}^{2}}$. The vertex reconstruction efficiency is sample dependent in fact nearby vertices can shadow a clean reconstruction. The expected vertex reconstruction efficiency is $\sim 95 \%$ for non-diffractive events.

An excellent vertex resolution has been achieved also for secondary vertices: from MC studies, for an hadronic interaction, the vertex resolution has been estimated to be $200-300 \mu \mathrm{m}$ (in both $R$ and $z$ ) for reconstructed vertices with radii $\leq 100 \mathrm{~mm}$ and $\sim 1 \mathrm{~mm}$ at larger radii [7]. Simulations have been used also to study higher pile-up scenarios.

\section{Material Studies}

The precise knowledge of the material budget within the tracking volume is mandatory for an accurate track reconstruction and a precise determination of the track parameters. Mapping of the amount of passive material in the tracking volume is mainly performed using photon conversions and hadronic interactions [7] (Fig. 5). The comparison between real data and simulation of the spatial distribution of the reconstructed secondary vertices (either with photon conversions or hadronic interactions) helps to improve the description of material in the detector geometry. In the same sense, the rate of photon conversions inside the tracker volume compared with the beryllium beam pipe (very well known object) helps to asses the amount of material inside the real detector. The material uncertainty in simulation is constrained summing photon conversions and hadronic interactions with other different techniques (e.g. study of $K^{0}$ and other mass signals, study of mul- 

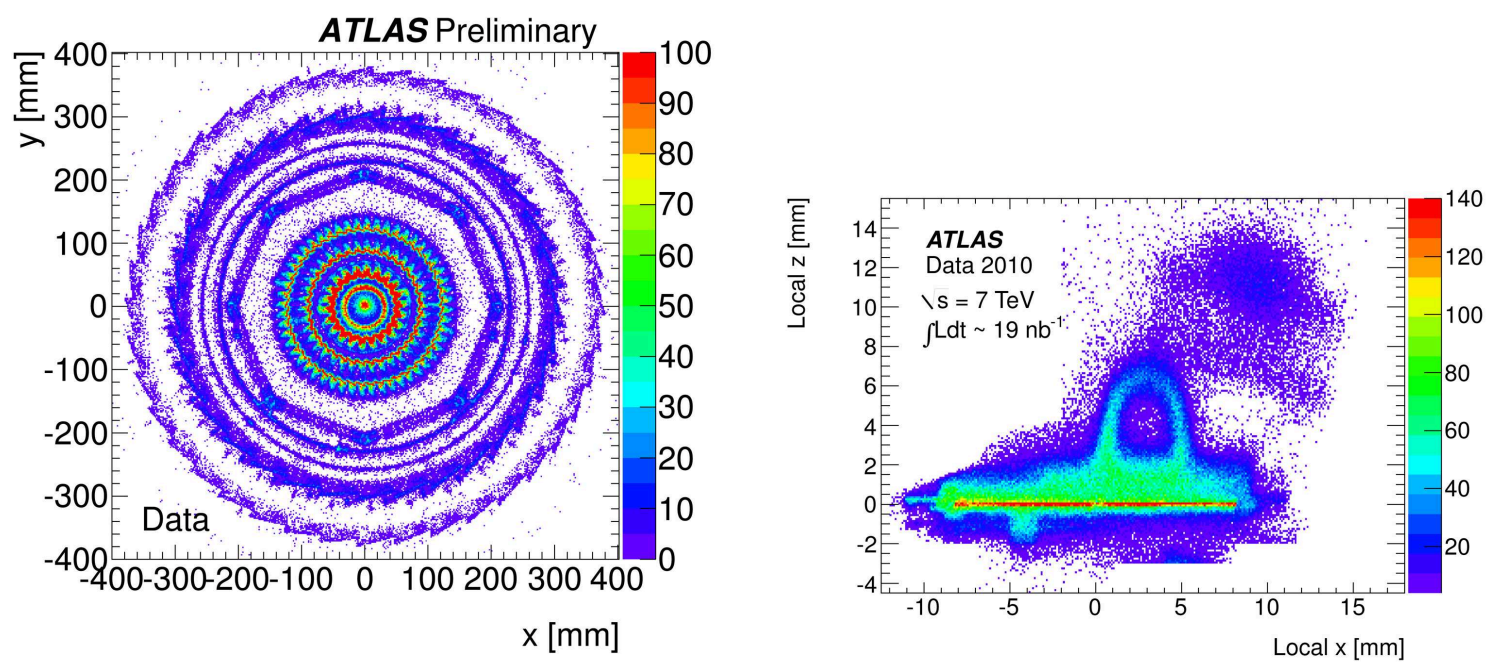

Figure 5: Distribution of the reconstructed photon conversions for data events as a function of the transverse positions $(x, y)$ (left), and of the reconstructed hadronic interactions for data events in the local coordinate system for the first pixel detector layer (right).

tiple scattering resolution term, etc.). The estimated uncertainty is better than $\sim 5 \%$ in the central region and at the level of $\sim 10 \%$ in the end-caps.

\section{Inner Detector Alignment}

The limited knowledge of the relative position of detector pieces should not lead to a significant degradation of the track parameter beyond the intrinsic tracker resolution, nor introduce biases. Thus the goal of the alignment is to provide an accurate description of the geometry of the detector: the location and orientation of every tracking element. A very high accuracy is needed for precision physics measurements, e.g. a $15 \mathrm{MeV}$ precision in $\mathrm{W}$ mass requires a $\sim 1 \mu \mathrm{m}$ alignment [2].

The ID has been aligned using a track based method [8]. It consists in a $\chi^{2}$ minimization of the track-hit residuals. The alignment procedure has been executed at 3 different levels in accordance with the assembly of the ID structures, i.e. with increasing number of aligned substructures proceeding from large structures to module level with increasing granularity of structures and degrees of freedom. The level 1 corresponds to the large barrel and end-cap structures of the Pixel, SCT and TRT (just 7 structures to align). The level 2 deals with the barrel layers and end-cap disks of the Pixel and SCT (31 structures) and the barrel modules and end-cap wheels of the TRT (176 structures). Finally, the level 3 aligns each pixel and SCT modules $(5,832)$ and TRT straw tubes (350k). In total, one has to deal with more than 700k degrees of freedom. The alignment is monitored on a run by run basis. There are two dedicated data streams selected by the high level trigger: a collection of high $\mathrm{p}_{T}$ and isolated collision tracks and cosmic-ray tracks triggered during the empty LHC bunches [9]. With a run-by-run alignment one can test the detector stability (Fig. 6 left). Large movements of the detector are due to changes in operational conditions (typical size $<10 \mu \mathrm{m})$ after hardware incidents. In between these periods little $(<1 \mu \mathrm{m})$ movement is observed indicating that the detector is generally very stable. In order to derive the alignment 

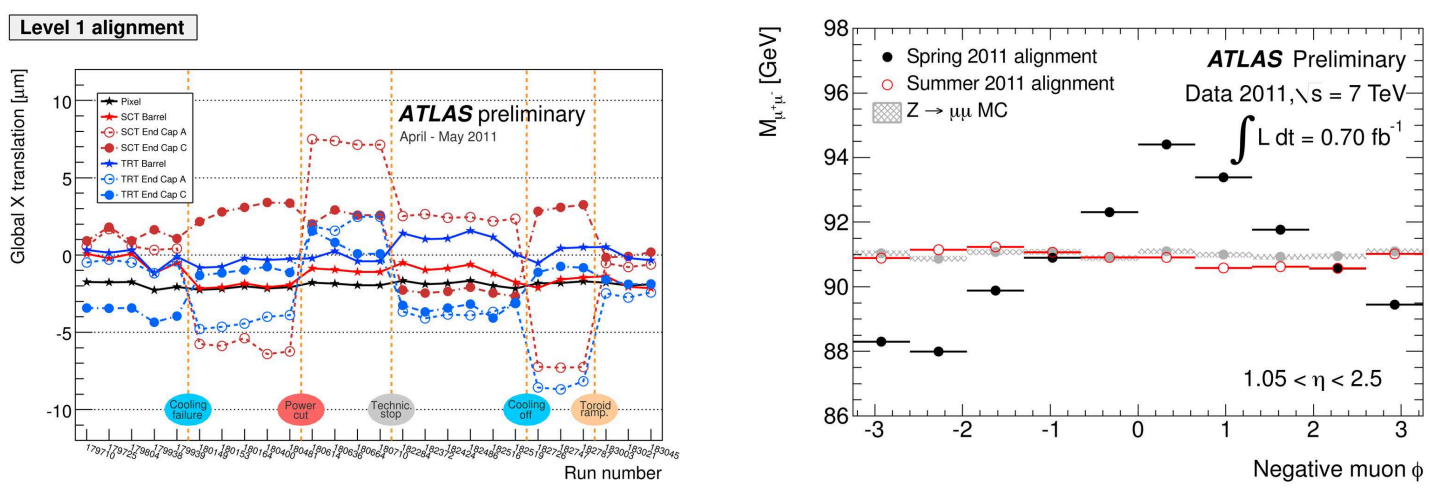

Figure 6: Subsystem level, Level One, alignment corrections performed on a run by run basis starting from a common set alignment constants (left), and mean $\mathrm{Z}$ invariant mass versus $\phi$ for negative muons (right).

constants over many data taking periods an offline alignment is run. The alignment software allows to set constraints on the beam spot, assembly survey data [10], momentum of the muons from the Muon Spectrometer and E/p from electrons.

A very detailed alignment validation program is executed, which comprises a detailed check of many alignment specific distributions (as the residuals of all components) and checks for track parameters and their errors. The resonance invariant masses (light as $K_{s}^{0}$ and heavy as $Z$ ) are scrutinized against all the track parameters in order to detect and correct possible biases (Fig. 6 right).

\section{7. $b$-tagging Performance}

The process of identifying jets originating from $b$-quark fragmentation ( $b$-jets) is called $b$ tagging. This has applications in many physics analyses, for example it greatly helps in the Standard Model measurements (e.g. $\sigma_{b \bar{b}}$, top physics, etc.), in the searches for the Higgs boson and for physics beyond the Standard Model.

The identification of $b$-jets exploits the high mass and relatively long lifetime of $b$-hadrons. They can fly a few millimeters before decaying. Jets containing $b$-hadrons can therefore be tagged either inclusively by measuring the impact parameters of the tracks (i.e. the distance of closest approach of the track to the collision point) of the $b$-hadron decay products, or by explicitly reconstructing a displaced vertices in jet. Furthermore the semi-leptonic decays of $b$-hadrons can also be used by tagging the lepton in the jet.

The first physics measurements published by the ATLAS Collaboration [11-13] used a set of basic algorithm termed as "early data taggers", which were commissioned shortly after the beginning of data taking [14]. More advanced taggers, with improved rejection capability have been recently calibrated [19] and already heavily used in ATLAS physics measurements.

\section{1 "early data taggers" and efficiency measurements}

For early 2010 data two simple $b$-tagging algorithms were commissioned: JetProb [15] and SV0 [16]. JetProb is an impact parameter based tagger using both the transverse and longitudinal impact parameters of tracks with respect to the primary vertex. It builds a variable giving a 
probability that all tracks in the jet originate from the primary vertex. The negative side of the distribution of the displacement (signed impact parameter significance $I P / \sigma_{I P}$ ) of tracks in a jet is symmetrized to get a calibration function that is used to calibrate the mentioned probability. On the other hand, in the SV0 tagger the secondary inclusive vertex formed by the decay products of the $b$-hadron, including products of the eventual subsequent $c$-hadron decay, is reconstructed. The signed decay length significance is used to discriminate $b$-jets against non- $b$-jets.

In order for $b$-tagging to be used in physics analyses, the efficiency with which a jet originating from a $b$-quark is tagged by a $b$-tagging algorithm needs to be measured, as well as the probability to tag a jet originating from a light-flavour quark, referred to as the mistag rate. The two early taggers were calibrated using several methods in ATLAS: a $50 \%$ and a $70 \% b$-tagging efficiency working points for JetProb (JetProb50 and JetProb70) and a 50\% $b$-tagging efficiency working point for SV0 (SV050). The average mistag rate is approximatively $0.5 \%$ for the high purity working point and $5 \%$ for the high efficiency working point [14].

One way to measure the $b$-tagging efficiency is by using muon-jets. Though muons originate also from other processes, a major source is the semi-leptonic decay of the $b$ - or $c$-quarks resulting from the $b$-quark decays. In the $p_{T}^{r e l}[14]$ measurement the momentum of a muon orthogonal to the flight axis of the jet it is associated to is used to measure the $b$-jet content of a given sample. Templates of $p_{T}^{r e l}$ for $b$ - , $c$ - and light-flavor jets are fit to the data before and after $b$-tagging and the efficiency is calculated as $\varepsilon=N_{b, t a g} / N_{b}$.

A similar measurement uses uncorrelated taggers to numerically calculate the $b$-tagging efficiency from a set of 8 equations (System8) [17]. System8 is designed to minimize the dependence on simulation and it is a very promising method that will be used in future $b$-tagging calibration results [18].

Another possibilty to calibrate the $b$-tagging efficiency is to select a physics process providing a sample of $b$-jet with high purity. Two channels are used in ATLAS:

- semi-leptonic decay chain $b \rightarrow D^{*} \mu X \rightarrow D^{0}(\rightarrow K \pi) \pi \mu X$ [14]. The mass reconstruction combined with the muon requirement yields a high $b$-jet purity and therefore gives direct access to the $b$-tagging efficiency.

- events with a top quark and an anti-top quark [14]. The top quark almost exclusively decays into a W-boson and a $b$-quark. Different methods to measure the $b$-tagging efficiency in a $t \bar{t}$ enriched sample have been developed and they all yield promising results that are becoming especially important as the integrated luminosity increases.

All of the described techniques have been used to measure the $b$-tagging efficiency in data. The results are all in good agreement with each other and the efficiency in data is very close to that obtained in simulated events as shown in Fig. 7.

\section{2 "advanced taggers"}

Advanced taggers use more pieces of information to tag $b$-jets than the early data taggers [19]. The IP3D high-performance tagging algorithm uses a likelihood ratio technique in which input variables are compared to pre-defined smoothed and normalized distributions for both the $b$ - and light-jet hypotheses, obtained from Monte Carlo simulation. The distributions in this case 


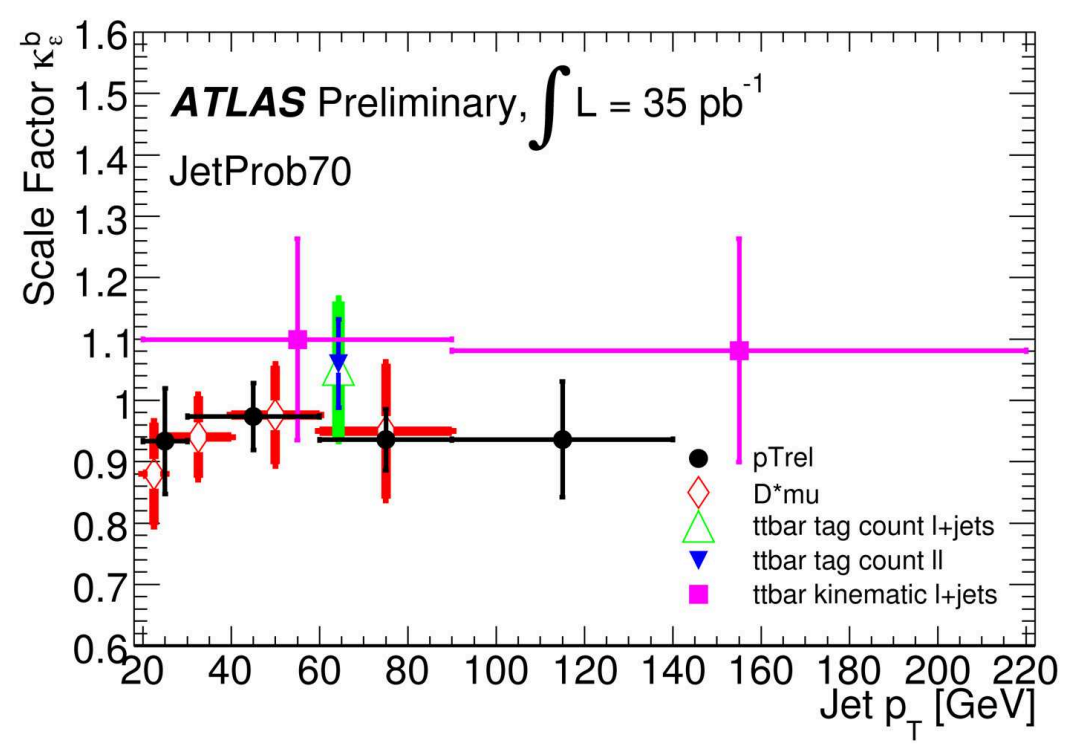

Figure 7: The measured b-tagging efficiency scale factors $k_{\varepsilon b}^{d a t a / s i m}$ for the $p_{T}^{r e l}, D^{*} \mu$ and $t \bar{t}$ methods for the JetProb70 algorithm.

are two-dimensional density functions of the signed transverse impact parameter significance and longitudinal impact parameter significance of tracks in jets, taking advantage of the correlations between the two variables.

To further increase the discrimination between $b$-jets and light jets, the inclusive vertex formed by the decay products of the $b$-hadron, including the products of the eventual subsequent charm hadron decay, can be sought (SV1 tagger). The decay length significance measured in three dimensions and signed with respect to the jet direction is used as a discriminating variable between $b$-jets and light jets, as well as three of the vertex properties: the invariant mass of all tracks associated to the vertex, the ratio of the sum of the energies of the tracks in the vertex to the sum of the energies of all tracks in the jet, and the number of two-track vertices. These variables are combined using a likelihood ratio technique.

Further discrimination can be achieved with another algorithm, called JetFitter, which exploits the topological structure of weak $b$ - and $c$-hadron decays inside the jet. A Kalman filter is used to find a common line on which the primary vertex and the $b$ - and $c$-vertices lie, as well as their position on this line, giving an approximated flight path for the $b$-hadron. With this approach, the $b$ - and $c$-hadron vertices are not necessarily merged, even when only a single track is attached to each of them. The discrimination between $b-, c$ - and light jets is based on a neutral network using similar variables as in the SV1 tagging algorithm above, and additional variables such as the flight length significances of the vertices.

Thanks to the likelihood ratio method used for IP3D and SV1, the algorithms can be easily combined by adding their respective weights. The combination JetFitter+IP3D is based on artificial neural network techniques with Monte Carlo simulated training samples and additional variables describing the topology of the decay chain. 

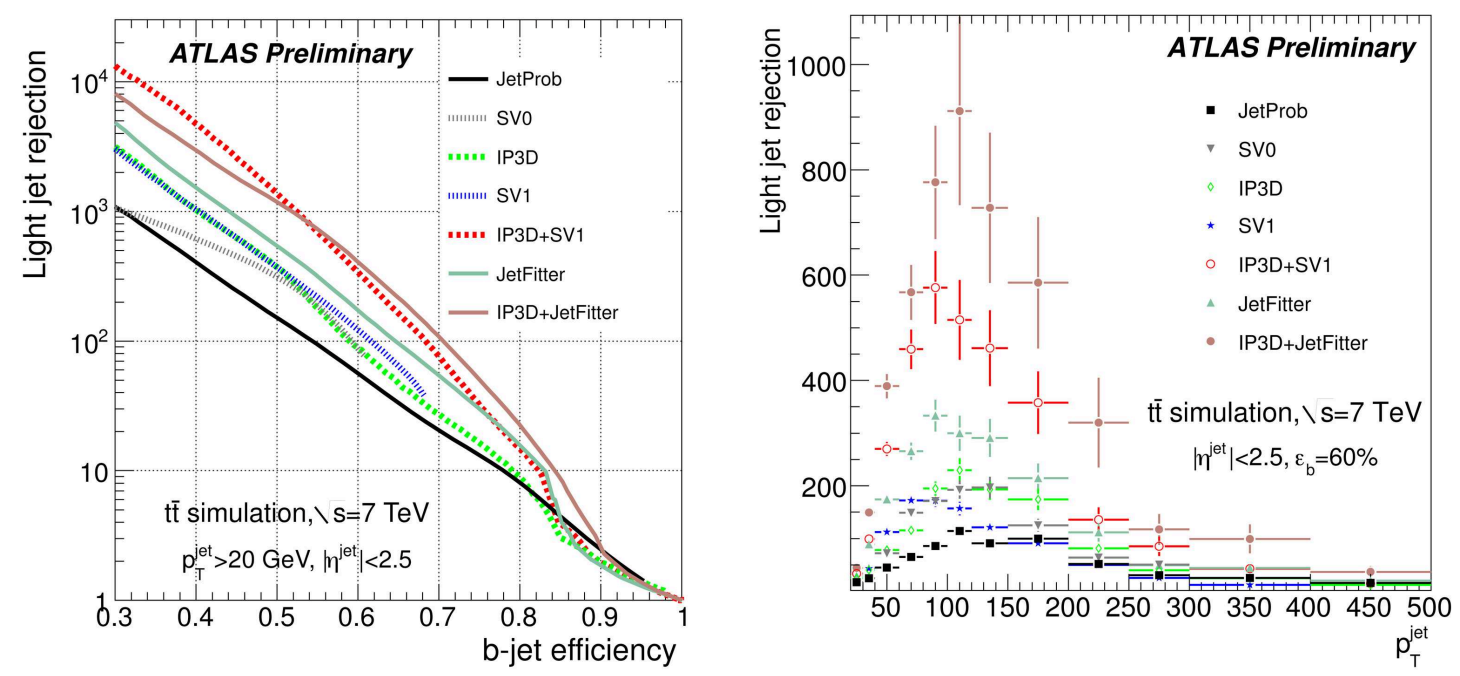

Figure 8: Light-jet rejection as a function of the $b$-jet tagging efficiency for the early tagging algorithms (JetProb and SV0) and for the high performance algorithms, based on simulated $t \bar{t}$ events (left), and lightjet rejection as a function of the jet transverse momentum $p_{T}$, for operating points of the various tagging algorithms leading to the same b-jet tagging efficiency of $60 \%$, based on simulated $t \bar{t}$ events (right).

The advanced taggers greatly improve light-jet rejection at a fixed $b$-tagging efficiency as is clearly visible in Fig.8. At same $b$-jet efficiency, the light jet rejection can be increased by a factor of 2 to 5 with new taggers allowing better background rejection. On the other hand for same light-jet rejection, the working point can be chosen at higher efficiency. This is very promising for searches with low production cross section.

\section{Conclusions}

The ATLAS Inner Detector tracking system performance has been discussed. The Pixels, SCT and TRT are operating very efficiently and providing high quality data for the physics analysis teams. The excellent performance of ATLAS track reconstruction and $b$-tagging satisfy the stringent requirements on Inner Detector track reconstruction to cover ATLAS physics program. The ID performance has reached its design level. Thanks to the very accurate vertex reconstruction the material of the detector has been mapped with high accuracy, and the aligned stability has been shown to be at the level of $<10 \mu \mathrm{m}$. 2010 heavy ion run as well gives good insights into tracking at high occupancy.

Several $b$-tagging algorithms have been developed and used in physics analyses. "Early taggers" operate at $50 \%$ efficiency with a background mistag rate of $0.5 \%$. High performance algorithms, providing a factor 2 to 5 improvement in light-jet rejection, have been commissioned and are already heavily used in ATLAS physics analyses.

\section{References}

[1] L. Evans and P. Bryant, LHC Machine, J. Instrum. 3, S08001 (2009). 
[2] ATLAS Collab., The ATLAS experiment at the CERN Large Hadron Collider, J. Instrum. 3, S08003 (2009).

[3] ATLAS Collab., Performance of the ATLAS Silicon Pattern Recognition Algorithm in Data and Simulation at $\sqrt{s}=7 \mathrm{TeV}$, ATLAS-CONF-2010-072, CERN, Geneva, 2010.

[4] ATLAS Collab., Measurement of underlying event characteristics using charged particles in $p p$ collisions at $\sqrt{s}=900 \mathrm{GeV}$ and $7 \mathrm{TeV}$ with the ATLAS detector, CERN-PH-EP-2010-063, CERN, Geneva, 2010.

[5] ATLAS Collab., Measurement of the centrality dependence of $J / \psi$ yields and observation of $Z$ production in lead-lead collisions with the ATLAS detector at the LHC, CERN-PH-EP-2011-146, CERN, Geneva, 2011.

[6] ATLAS Collab., Performance of primary vertex reconstruction in proton-proton collisions at $\sqrt{s}=7 \mathrm{TeV}$ in the ATLAS experiment, ATLAS-CONF-2010-069, CERN, Geneva, 2010.

[7] ATLAS Collab., Mapping the material in the ATLAS Inner Detector using secondary hadronic interactions in 7 TeV collisions, ATLAS-CONF-2010-058, CERN, Geneva, 2010.

[8] ATLAS Collab., Alignment of the ATLAS Inner Detector Tracking System with 2010 LHC proton-proton collisions at $\sqrt{s}=7 \mathrm{TeV}$, ATLAS-CONF-2011-012, CERN, Geneva, 2011.

[9] ATLAS Collab., The ATLAS Inner Detector commissioning and calibration, CERN-PH-EP-2010-043, CERN, Geneva, 2010.

[10] A. Andreazza, V. Kostyukhin and R. J. Madaras, Survey of the ATLAS Pixel Detector Components, ATL-INDET-PUB-2008-012, CERN, Geneva, 2008.

[11] ATLAS Collab., Measurement of the cross section for the production of a $W$ boson in association with b-jets in pp collisions at $\sqrt{s}=7 \mathrm{TeV}$ with the ATLAS detector, CERN-PH-EP-2011-132, CERN, Geneva, 2011.

[12] ATLAS Collab., Measurement of the top quark pair production cross section in pp collisions at $\sqrt{s}=7 \mathrm{TeV}$ in dilepton final states with ATLAS, CERN-PH-EP-2011-103, CERN, Geneva, 2011.

[13] ATLAS Collab., Measurement of the inclusive and dijet cross-sections of b-jets in pp collisions at $\sqrt{s}=7$ TeV with the ATLAS detector, CERN-PH-EP-2010-090, CERN, Geneva, 2010.

[14] ATLAS Collab., Calibrating the b-Tag Efficiency and Mistag Rate in $35 \mathrm{pb}^{-1}$ of Data with the ATLAS Detector, ATLAS-CONF-2011-089, CERN, Geneva, 2011.

[15] ATLAS Collab., Impact parameter-based b-tagging algorithms in the $\sqrt{s}=7$ TeV collision data with the ATLAS detector: the TrackCounting and JetProb algorithms, ATLAS-CONF-2010-041, CERN, Geneva, 2010.

[16] ATLAS Collab., Performance of the ATLAS Secondary Vertex b-tagging Algorithm in $\sqrt{s}=7 \mathrm{TeV}$ Collision Data, ATLAS-CONF-2010-042, CERN, Geneva, 2010.

[17] ATLAS Collab., Expected Performance of the ATLAS Experiment Detector, Trigger and Physics, CERN-OPEN-2008-020, CERN, Geneva, 2008.

[18] ATLAS Collab., b-Jet Tagging Efficiency Calibration using the System8 Method, ATLAS-CONF-2011-143, CERN, Geneva, 2011.

[19] ATLAS Collab., Commissioning of the ATLAS high-performance b-tagging algorithms in the $7 \mathrm{TeV}$ collision data, ATLAS-CONF-2011-102, CERN, Geneva, 2011. 